\title{
Quasiconformal Harmonic Mappings Related to Janowski Starlike Functions
}

(Pemetaan Harmonik Kuasimensebentuk Berkaitan dengan Fungsi Bakbintang Janowski)

\section{YASEMIN KAHRAMANER* \& YAŞAR POLATOĞLU}

\section{ABSTRACT}

Let $f(z)=h(z)+\overline{g(z)}$ be a univalent sense-preserving harmonic mapping of the open unit disc $D=\{z|| z \mid<1\}$.Iff satisfies the condition $|\omega(z)|=\left|\frac{g^{\prime}(z)}{h^{\prime}(z)}\right|<k, 0<k<1$, then is called $k$-quasiconformal harmonic mapping in D. The main purpose of this paper was to give some properties of the class of $k$-quasiconformal mappings related to Janowski starlike functions.

Keywords: Coefficient inequality; distortion theorem; growth theorem; $k$-quasiconformal mapping

\section{ABSTRAK}

Andaikan $f(z)=h(z)+\overline{g(z)}$ pemetaan harmonik terawet univalen bagi cakera unit terbuka $D=\{z|| z \mid<1\}$. Jika $f$ memenuhi syarat $|\omega(z)|=\left|\frac{g^{\prime}(z)}{h^{\prime}(z)}\right|<k, 0<k<1$, maka f dipanggil pemetaan harmonik $k$-kuasimensebentuk dalam D. Tujuan utama kertas ini adalah untuk memberi beberapa sifat bagi kelas pemetaan k-kuasimensebentuk yang berkaitan dengan fungsi bakbintang Janowski.

Kata kunci: Ketaksamaan pekali; pemetaan k-kuasimensebentuk; teorem erotan; teorem pertumbuhan

\section{INTRODUCTION}

Let be the family of functions regular in $D$ and satisfying the conditions $\phi(0)=0,|\phi(z)|<1$ for all $z \in D$.

Next, for arbitrary fixed numbers $A, B,-1<A \leq 1,-1$ $B<\mathrm{A}$, denote by $P(A, B)$, the family of functions $p(z)=1$ $+p_{1} z+p_{2} z^{2}+\ldots$ regular in $D$ and such that $p(z)$ is in $P(A$, $B)$ if and only if:

$$
p(z)=\frac{1+A \phi(z)}{1+B \phi(z)}
$$

for some $\phi(z) \in \Omega$ and every $z \in D$.

Moreover, let $S^{*}(A, B)$ denote the family of functions $h(z)=z+c_{2} z^{2}+\ldots$ regular in $D$ and such that $h(z)$ is in $S^{*}(A, B)$ if and only if

$$
z \frac{h^{\prime}(z)}{h(z)}=p(z)
$$

for some $p(z) \in P(A, B)$ and $z \in \mathrm{D}$. Let $s_{1}(z)=z+d_{2} z^{2}+$ $\ldots$ and $s_{2}(z)=z+e_{2} z^{2}+\ldots$ be analytic functions in the open unit $\operatorname{disc} D$. If there exists a function $\phi(z) \in \Omega$ such that $s_{1}(z)=s_{2}(\phi(z))$ for all $z \in D$, then we say that $s_{1}(z)$ is subordinate to $s_{2}(z)$ and we write $s_{1}(z) \prec s_{2}(z)$. Specially if $s_{2}(z)$ is univalent in $D$, then $s_{1}(z) \prec s_{2}(z)$ if and only if $s_{1}(D) \subset s_{2}(D)$ implies $s_{1}\left(D_{r}\right) \subset s_{2}\left(D_{r}\right)$, where $D_{r}=\{z|| z \mid$ $<r<1\}$ (Duren 1983; Goodman 1983).

Finally, a planar harmonic mapping in the open unit disc $D$ is a complex-valued harmonic function $f(z)$ which maps $D$ onto the some planar domain $f(D)$. Since
$D$ is a simply connected domain, the mapping $f(z)$ has a canonical decomposition $f(z)=h(z)+\overline{g(z)}$, where $h(z)$ and $g(z)$ are analytic in $D$ and have the following power series expansion:

$$
\begin{aligned}
& h(z)=\sum_{n=0}^{\infty} a_{n} z^{n} \\
& g(z)=\sum_{n=0}^{\infty} b_{n} z^{n},
\end{aligned}
$$

where $a_{n}, b_{n} \in C, n=0,1,2, \ldots$, as usual we call the analytic part of $h(z)$ and $g(z)$ is co-analytic part of $f(z)$. An elegant and complete treatment theory of the harmonic mapping is given in Duren's monograph (Duren 2004).

For this investigation we will need the following theorem and lemma:

Theorem 1 (Janowski 1973) Let be an element of $S^{*}(A, B)$, then

$$
C(r,-A,-B) \leq|h(z)| \leq C(r, A, B),
$$

where

$$
C(r, A, B)=\left\{\begin{aligned}
r(1+B r)^{\frac{A-B}{B}}, & B \neq 0 \\
r e^{A r} & , B=0
\end{aligned}\right.
$$

These bounds are sharp, being attained at the point $z=r e^{i \theta}, 0 \leq \theta \leq 2 \pi$ by 


$$
\begin{aligned}
& f_{*}=f_{0}(z,-A,-B), \\
& f^{*}=z f_{0}(z, A, B),
\end{aligned}
$$

respectively, where

$$
f_{0}(z)=\left\{\begin{array}{c}
\left(1+B e^{-i \theta} z\right)^{\frac{A-B}{B}}, \quad B \neq 0 \\
r e^{A e^{-i \theta}}, \quad B=0 .
\end{array}\right.
$$

Lemma 1 (Jack 1971) Let $\phi(z)$ be regular in the unit disk $D$ with $\phi(0)$. Then, if $|\phi(z)|$ attains its maximum value on the circle $|z|=r$ at the point $z_{1}$, one has $z_{1} \phi^{\prime}\left(z_{1}\right)=k \phi\left(z_{1}\right)$, for $k \geq 1$.

Lewys (1936) proved that the harmonic function $f(z)$ is locally univalent in $D$ if and only if its Jacobian

$$
J_{f}=\left|h^{\prime}(z)\right|-\left|g^{\prime}(z)\right|,
$$

is different from zero in $D$. In the view of this result, locally univalent harmonic mappings in the open unit disc $D$ are either sense-preserving if $\left|h^{\prime}(z)\right|>\left|g^{\prime}(z)\right|$ in $D$ or sensereversing if $\left|g^{\prime}(z)\right|>\left|h^{\prime}(z)\right|$ in $D$. Throughout this paper, we will restrict ourselves to the study of sense-preserving harmonic mappings. We also note that $f(z)=h(z)+\overline{g(z)}$ is sense-preserving in $D$ if and only if $h^{\prime}(z)$ does not vanish in $D$ and the second dilatation $|\omega(z)|=\mid \frac{g^{\prime}(z)}{h^{\prime}(z)}$ has the property $|\omega(z)|<1$ for all $z \in D$. Therefore, the class of all sensepreserving harmonic mappings in the open unit disc $D$ with $a_{0}=b_{0}=0$ and $a_{1}=1$ will be denoted by $S_{H}$. Thus, $S_{H}$ contains the standard class $S$ of univalent functions. The family of all mappings $f \in S_{H}$ with the additional property $g^{\prime}(0)=0$, i.e., $b_{1}=0$ is denoted by $S_{H}^{0}$. Hence it is clear that $S \subset S_{H}^{0} \subset S_{H}$. A univalent harmonic mapping is called $k$-quasiconformal $(0<k<1)$ if $|\omega(z)|<k$. For the general definition of quasiconformal mapping, see Duren (2004).

The main purpose of this paper was to investigate the class of the $k$-quasiconformal harmonic mappings

$$
\begin{aligned}
S_{H}^{*}(k q, A, B)= & \left\{f(z)=h(z)+\overline{g(z)} \in S_{H}|| \omega(z) \mid\right. \\
& \left.<k, 0<k<1, h(z) \in S^{*}(A, B)\right\} .
\end{aligned}
$$

\section{MAIN RESULTS}

Theorem 2 Let $f(z)=h(z)+\overline{g(z)}$ be an element of $S_{H}^{*}(k q$, $A, B)$ then

$$
\frac{g^{\prime}(z)}{h^{\prime}(z)} \prec \frac{k^{2}\left(b_{1}-z\right)}{k^{2}-\overline{b_{1}} z} .
$$

Proof We consider the transformation $\frac{k^{2}\left(b_{1}-z\right)}{k^{2}-b_{1} z}$. This transformation maps $|z|<k$ onto itself. On the other hand, we have:

$$
\omega(z)=\frac{g^{\prime}(z)}{h^{\prime}(z)}=\frac{\left(b_{1} z+b_{2} z^{2}+\ldots\right)^{\prime}}{\left(z+a_{2} z^{2}+\ldots\right)^{\prime}}=\frac{b_{1}+2 b_{2} z+\ldots}{1+2 a_{2} z+\ldots} \Rightarrow \omega(0)=b_{1} .
$$

Therefore, the function

$$
\phi(z)=\frac{k^{2}\left(b_{1}-\omega(z)\right)}{k^{2}-\bar{b}_{1} \omega(z)},
$$

satisfies the conditions of Schwarz lemma, then we have

$$
\omega(z)=\frac{k^{2}\left(b_{1}-\phi(z)\right)}{k^{2}-\overline{b_{1}} \phi(z)} \Rightarrow \omega(z)=\frac{g^{\prime}(z)}{h^{\prime}(z)} \prec \frac{k^{2}\left(b_{1}-z\right)}{k^{2}-\overline{b_{1}} z} .
$$

On the other hand, the transformation $\frac{k^{2}\left(b_{1}-z\right)}{k^{2}-\overline{b_{1}} z}$ maps $|z|=r$ onto the disc with the centre

$$
C(r)=\left(\frac{k^{2}\left(1-r^{2}\right) \operatorname{Re} b_{1}}{k^{2}-\left|b_{1}\right|^{2} r^{2}}, \frac{k^{2}\left(1-r^{2}\right) \operatorname{Im} b_{1}}{k^{2}-\left|b_{1}\right|^{2} r^{2}}\right),
$$

and the radius

$$
\rho(r)=\frac{k\left(k^{2}-\left|b_{1}\right|^{2}\right) r}{k^{2}-\left|b_{1}\right|^{2} r^{2}} \text {. }
$$

Then, we write:

$$
\omega\left(D_{r}\right)=\left\{z_{1}\left|\omega(z)-\frac{k^{2}\left(1-r^{2}\right) b_{1}}{k^{2}-\left|b_{1}\right|^{2} r^{2}}\right| \leq \frac{k\left(k^{2}-\left|b_{1}\right|^{2}\right) r}{k^{2}-\left|b_{1}\right|^{2} r^{2}}\right\} .
$$

Now we define the function $\phi(z)$ by:

$$
\frac{g(z)}{h(z)}=\frac{k^{2}\left(b_{1}-\phi(z)\right)}{k^{2}-\overline{b_{1}} \phi(z)} .
$$

Then, $\phi(z)$ is analytic and $\phi(0)=\frac{k^{2} .0}{\left|b_{1}\right|^{-}-k^{2}}=0$. If we take the derivative from (4) and after the brief calculations we get

$$
\omega(z)=\frac{g^{\prime}(z)}{h^{\prime}(z)}=\frac{k^{2}\left(b_{1}-\phi(z)\right)}{k^{2}-\overline{b_{1}} \phi(z)}+\frac{k^{2}\left(\left|b_{1}\right|^{2}+k^{2}-2 b_{1} \phi(z)\right) \phi^{\prime}(z)}{\left(k^{2}-\bar{b}_{1} \phi(z)\right)^{2}} \cdot \frac{h(z)}{h^{\prime}(z)} .
$$

On the other hand, since $h(z) \in S^{*}(A, B)$, then the boundary value of $z \frac{h(z)}{h^{\prime}(z)}$ is

$$
\frac{\left(1-A B r^{2}\right)+(A-B) r e^{i \theta}}{1-B^{2} r^{2}} \text {. }
$$

Therefore, (5) can be written in the following form on $|z|=r$ :

$$
\omega(z)=\frac{\left.k^{2}\left(b_{1}-\phi(z)\right)\right)}{k^{2}-\bar{b}_{1} \phi(z)}+\frac{k^{2}\left(\left|b_{1}\right|^{2}+k^{2}-2 b_{1} \phi(z)\right) \mid \phi^{\prime}(z)}{\left(k^{2}-\overline{b_{1}} \phi(z)\right)^{2}} \cdot \frac{1-B^{2} r^{2}}{\left(1-A B r^{2}\right)+(A-B) r^{i \theta}} .
$$

In this step if we use Jack lemma, then (6) can be written in the following form:

$$
\begin{aligned}
& \omega\left(z_{1}\right)=\frac{g^{\prime}\left(z_{1}\right)}{h^{\prime}\left(z_{1}\right)}=\frac{k^{2}\left(b_{1}-\phi\left(z_{1}\right)\right)}{k^{2}-\vec{b}_{1} \phi\left(z_{1}\right)}+\frac{k^{2}\left(\left|b_{1}\right|^{2}+k^{2}-2 b_{1} \phi\left(z_{1}\right)\right) k \phi^{\prime}\left(z_{1}\right)}{\left(k^{2}-b_{1} \phi\left(z_{1}\right)\right)^{2}} \\
& \frac{1-B^{2} r^{2}}{\left(1-A B r^{2}\right)+(A-B) r e^{i \theta}} \notin \omega\left(D_{r}\right)
\end{aligned}
$$


But this contradicts to (3) because $\left|\phi\left(z_{1}\right)\right|=1, k \geq 1$ so our assumption is wrong, i.e., $|\phi(z)|<1$ for all $z \in D$. Therefore, (4) shows that

$$
\frac{g(z)}{h(z)} \prec \frac{k^{2}\left(b_{1}-z\right)}{k^{2}-\overline{b_{1}} z}
$$

Corollary 1 Let $f(z)=h(z)+\overline{g(z)}$ be an element of $S_{H}^{*}(k q$, $A, B)$, then

$$
\left.\begin{array}{c}
\frac{k\left(b_{1} \mid-k r\right)}{k-b_{1} \mid r} F(A, B,-r) \leq\left|g^{\prime}(z)\right| \leq \frac{k\left(\left|b_{1}\right|+k r\right)}{k+\left|b_{1}\right| r} F(A, B, r), B \neq 0 \\
\frac{k\left(\left|b_{1}\right|-k r\right)}{k-b_{1} \mid r} F_{1}(A,-r) \leq\left|g^{\prime}(z)\right| \leq \frac{k\left(b_{1} \mid+k r\right)}{k+b_{1} \mid r} F_{1}(A, r), B=0
\end{array}\right\}
$$

where

$$
\begin{aligned}
& F(A, B, r)=(1+A r)(1+B r)^{\frac{A-2 B}{B}}, \\
& F_{1}(A, r)=(1+A r) e^{A r} .
\end{aligned}
$$

and

$$
\frac{k\left(b_{1} \mid-k r\right)}{k-b_{1} \mid r} C(-A,-B, r) \leq|g(z)| \leq \frac{k\left(b_{1} \mid+k r\right)}{k+\left|b_{1}\right| r} C(A, B, r)
$$

where

$$
C(A, B, r)=\left\{\begin{array}{c}
r(1+B r)^{\frac{A-B}{B}}, B \neq 0 \\
r e^{A r}, B=0
\end{array} .\right.
$$

Proof Since we have

$$
\begin{gathered}
\left|z \frac{h^{\prime}(z)}{h(z)}-\frac{1-A B r^{2}}{1-B^{2} r^{2}}\right| \leq \frac{(A-B) r}{1-B^{2} r^{2}} \Rightarrow \frac{1-A r}{1-B r} \leq\left|z \frac{h^{\prime}(z)}{h(z)}\right| \leq \frac{1+A r}{1+B r} \\
\left.\begin{array}{c}
\frac{1}{r} \cdot \frac{1-A r}{1-B r}|h(z)| \leq\left|h^{\prime}(z)\right| \leq \frac{1}{r} \cdot \frac{1+A r}{1+B r}|h(z)|, B \neq 0 \\
\frac{1}{r} \cdot(1-A r)|h(z)| \leq\left|h^{\prime}(z)\right| \leq \frac{1}{r} .(1+A r)|h(z)|, B=0
\end{array}\right\} .
\end{gathered}
$$

Using Theorem 2, from (9) we obtain that

$$
\left.\begin{array}{c}
F(A, B,-r) \leq\left|h^{\prime}(z)\right| \leq F(A, B, r) \\
F_{1}(A,-r) \leq\left|h^{\prime}(z)\right| \leq F_{1}(A, r)
\end{array}\right\},
$$

where

$$
\begin{gathered}
F(A, B, r)=(1+A r)(1+B r)^{\frac{A-2 B}{B}}, \\
F_{1}(A, r)=(1+A r) e^{A r} .
\end{gathered}
$$

On the other hand, a simple consequence of Theorem 2 follows:

$$
\left.\begin{array}{l}
\frac{k\left(b_{1} \mid-k r\right)}{k-b_{1} \mid r}\left|h^{\prime}(z)\right| \leq\left|g^{\prime}(z)\right| \leq \frac{k\left(\left|b_{1}\right|+k r\right)}{k+\left|b_{1}\right| r}\left|h^{\prime}(z)\right| \\
\frac{k\left(\left|b_{1}\right|-k r\right)}{k-b_{1} \mid r}|h(z)| \leq|g(z)| \leq \frac{k\left(b_{b} \mid+k r\right)}{k+b_{1} \mid r}|h(z)|
\end{array}\right\} .
$$

By considering Theorem 2, (10) and (11) we get (7) and (8).
Corollary 2 Let $f(z)=h(z)+\overline{g(z)}$ be an element of $S_{H}^{*}(k q$, $A, B)$ then

$$
\left.\begin{array}{c}
(F(A, B,-r))^{2} \frac{k^{2}\left(\left|b_{1}\right|-k r\right)^{2}}{\left(k-\left|b_{1}\right| r\right)^{2}} \leq J_{f} \leq(F(A, B, r))^{2} \frac{k^{2}\left(\left|b_{1}\right|+k r\right)^{2}}{\left(k+\left|b_{1}\right| r\right)^{2}}, B \neq 0 \\
\left(F_{1}(A,-r)\right)^{2} \frac{k^{2}\left(\left|b_{1}\right|-k r\right)^{2}}{\left(k-\left|b_{1}\right| r\right)^{2}} \leq J_{f} \leq\left(F_{1}(A, r)\right)^{2} \frac{k^{2}\left(\left|b_{1}\right|+k r\right)^{2}}{\left(k+\left|b_{1}\right| r\right)^{2}}, B=0
\end{array}\right\}
$$

Proof This corollary is a simple consequence of the Corollary 1 and the definition of the Jacobian of $f$.

Corollary 3 Let $f(z)=h(z)+\overline{g(z)}$ be an element of $S_{H}^{*}(k q$, $A, B$ ) then

$$
\left.\begin{array}{c}
\int F(A, B,-r) \frac{k\left(1+b_{1} \mid\right)+\left(\left|b_{1}\right|-k^{2}\right) r}{k+b_{b} \mid r} d r \leq|f| \leq \int F(A, B, r) \frac{k\left(1+b_{1} \mid\right)+\left(\left|b_{1}\right|+k^{2}\right) r}{k+\left|b_{1}\right| r} d r, B \neq 0 \\
\int F(A, B,-r) \frac{k\left(1+b_{1} \mid\right)+\left(\left|b_{1}\right|-k^{2}\right) r}{k+\left|b_{1}\right| r} d r \leq|f| \leq \int F_{1}(A, r) \frac{k\left(1+b_{1} \mid\right)+\left(b_{1} \mid+k^{2}\right) r}{k+b_{1} \mid r} d r, B=0
\end{array}\right\}
$$

Proof Using (3) we get

$$
\begin{aligned}
& \frac{k\left(1-b_{1} \mid\right)+\mid\left(b_{1} \mid-k^{2}\right) r}{k+b_{1} \mid r} \leq 1-|\omega(z)| \leq \frac{k\left(1-b_{1} \mid\right)-\left(\left|b_{1}\right|-k^{2}\right) r}{k-b_{b} \mid r}, \\
& \frac{k\left(1+\left|b_{1}\right|\right)-\left(\left|b_{1}\right|+k^{2}\right) r}{k-\left|b_{1}\right| r} \leq 1+|\omega(z)| \leq \frac{k\left(1+b_{1} \mid\right)+\left(\left|b_{1}\right|+k^{2}\right) r}{k+b_{1} \mid r} .
\end{aligned}
$$

On the other hand,

$$
\begin{aligned}
& \left(\left|h^{\prime}(z)\right|\right)-\left(\left|g^{\prime}(z)\right|\right)|d z| \leq|d f| \leq\left(\left|h^{\prime}(z)\right|+\left|g^{\prime}(z)\right|\right)|d z| \\
& \Rightarrow\left|h^{\prime}(z)\right|(1-|\omega(z)|)|d z| \leq|d f| \leq\left|h^{\prime}(z)\right|(1+|\omega(z)|)|d z| .
\end{aligned}
$$

Using (9), (12) and (13) in (14) and integrating, we obtain the desired result.

Theorem 3 Let $f(z)=h(z)+\overline{g(z)}$ be an element of $S_{H}^{*}(k q$, $A, B)$ then

$$
\sum_{m=2}^{n} k^{4}\left|b_{m}-b_{1} a_{m}\right|^{2} \leq\left(\left|b_{1}\right|^{2}-k^{2}\right)^{2}+\sum_{m=2}^{n}\left|\overline{b_{1}} b_{m}-k^{2} a_{m}\right|^{2} .
$$

Proof Using Theorem 2 then we can write:

$$
\begin{aligned}
& \frac{g(z)}{h(z)} \frac{k^{2}\left(b_{1}-\phi(z)\right)}{k^{2}-\overline{b_{1}} \phi(z)} \Leftrightarrow k^{2} g(z)-k^{2} b_{1} h(z) \\
& =\left(\overline{b_{1}} g(z)-k^{2} h(z)\right) \phi(z) .
\end{aligned}
$$

Therefore, we have

$$
\begin{aligned}
& \sum_{m=2}^{n}\left(k^{2} b_{m}-k^{2} b_{1} a_{m}\right) z^{m}+\sum_{m=n+1}^{\infty} d_{m} z^{m} \leq \\
& {\left[\left(\left|b_{1}\right|^{2}-k^{2}\right) z+\sum_{m=2}^{n}\left(\bar{b}_{1} b_{m}-k^{2} a_{m}\right)\right] \phi(z),}
\end{aligned}
$$

where the coefficients $d_{m}$ have been chosen suitably and (16) can be written in the form

$$
|F(z)=G(z) \phi(z), \quad| \phi(z) \mid<1,
$$


then we have:

$$
\begin{aligned}
& |F(z)|^{2}=|G(z) \phi(z)|^{2}=|G(z)|^{2}|\phi(z)|^{2}<\left.G(z)\right|^{2} \\
& \Rightarrow \frac{1}{2 \pi} \int_{0}^{2 \pi}\left|F\left(r e^{i \theta}\right)\right|^{2} d \theta \leq \frac{1}{2 \pi} \int_{0}^{2 \pi}\left|G\left(r e^{i \theta}\right)\right|^{2} d \theta \\
& \Rightarrow \sum_{m=2}^{n} k^{4}\left|b_{m}-b_{1} a_{m}\right|^{2} r^{2 k}+\sum_{m=m+1}^{\infty}\left|d_{m}\right|^{2} r^{2 k} \\
& \leq\left[\left(\left|b_{1}\right|^{2}-k^{2}\right)^{2} r^{2 k}+\sum_{m=1}^{n}\left|\overline{b_{1}} b_{m}+k^{2} a_{m}\right|^{2} r^{2 k}\right],
\end{aligned}
$$

passing to the limit as $r \rightarrow 1$ we obtain (15). The method of this proof was based on the Clunie method (Clunie 1959).

\section{CONCLUSION}

In this paper, the class of the quasiconformal harmonic mappings related to the Janowski starlike functions was investigated. In this investigation, we have derived the basic characterization, distortion theorem, growth theorem and the coefficient inequality of the mentioned class.

\section{REFERENCES}

Clunie, J. 1959. On Meromorphic schlicht functions. J. London Math. Soc. 34: 215-216.

Duren, P. 2004. Harmonic Mappings in the Plane. Vol. 156 of Cambridge Tracts in Mathematics. Cambridge: Cambridge University Press.
Duren, P. 1983. Univalent Functions. Berlin: Springer Verlag. Goodman, A.W. 1983. Univalent Functions. Volume I. Tampa Florida: Mariner Publishing Company Inc.

Jack, I.S. 1971. Functions starlike and convex of order alpha. $J$. London Math. Soc. 3: 369-374.

Janowski, W. 1973. Some extremal problems for certain families of analytic functions I. Annales Policini Mathematici 28: 297-326.

Lewys, H. 1936. On the non-vanishing of the Jacobian in certain one-to-one mappings. Bull. Amer. Math. Soc. 42: 689-692.

Yasemin Kahramaner*

Department of Mathematics

Istanbul Commerce University, İstanbul

Turkey

Yaşar Polatoğlu

Department of Mathematics and Computer Science

İstanbul Kültür Üniversitesi, İstanbul

Turkey

*Corresponding author; email: ykahramaner@ticaret.edu.tr

Received: 17 December 2013

Accepted: 8 July 2014 